\title{
Mais um tributo às análises editoriais
}

\author{
Harley E. A. Bicas; Cristina Muccioli; Mauro S. O. Campos; Mauro Goldchmit; Samir J. Bechara; Vital Paulino Costa
}

No ano 2000, nossa redação recebeu o encaminhamento de 194 trabalhos (exclusive os de Atualização Continuada e outras matérias), para cada um dos quais foram sempre designados dois analistas editoriais, muito freqüentemente três ou, em algumas vezes, quatro (quando discordâncias substanciais nas recomendações sobre as publicações nos são enviadas). Isso requer cerca de 450 pareceres. Por outro lado, são 69 os membros listados como de nosso Conselho Editorial o que daria, em média, cerca de 6,4 análises para cada um, no período de doze meses. Uns, aliás, por natureza de suas "especializações" seriam menos acionados, cabendo, portanto, mais serviços aos outros. Acresça-se ainda que todos, já muito atarefados por suas várias ocupações vêem-se, não raramente, assoberbados por novos compromissos, obrigando-se a solicitar, temporariamente, aquilo que, na verdade, lhes seria de direito: umas "férias". O resultado é óbvio: muitos desses Conselheiros teriam que receber, quase todo mês, trabalhos para emissão de pareceres.

É, portanto, quase uma imposição matemática a necessidade de termos de recorrer a consultores adicionais. E, felizmente, já podemos contar com um apreciável contingente de pessoas muito bem qualificadas para essas atribuições. Essa condição nos permite conservar a qualidade dos Arquivos Brasileiros de Oftalmologia com um padrão mais alto, depurando os artigos enviados de suas imperfeições maiores, melhorando apresentações e o aproveitamento de seus próprios dados, aconselhando complementações àqueles ainda estruturalmente debilitados, evitando que exposições insatisfatórias possam comprometer o prestígio de seus autores. É um trabalho silencioso, mas indispensável; e tão importante que seu anonimato chega, às vezes, a ser profundamente injusto. Tanto que estamos oferecendo (desde os dois últimos números) a oportunidade de que os revisores se declarem como tal. Em vários casos, contribuem em questões fundamentais e de conteúdo, em outros com alterações de apresentação e forma; ou, então, agem, pelo menos, como avalistas da publicação, como se fizessem parte da equipe de seus autores. Claro que muitos dos analistas preferem que seus nomes não sejam declarados. Mas, por um melhor entendimento do serviço prestado pelos analistas editoriais, o que parece vir se tornando cada vez mais sentido em nosso meio, é possível que os próprios autores sejam os primeiros a reconhecer a vantagem e a conveniência dessa tarefa desempenhada.

Temos considerado os analistas editoriais "anjos da guarda" dos autores, guiando-os, aconselhando-os, evitando-lhes situações embaraçosas. Em muitos casos, é im- pressionante e inequívoca a contribuição de melhoria suscitada por essas pessoas: comparando-se um artigo em sua forma original e na que vai à publicação, quase não se pode reconhecer a identidade entre elas. Às vezes, nem sequer no próprio título.

Isso tudo tem um custo: maior demora para que a matéria venha à luz; esforços dos autores naquilo que, inicialmente, consideravam um trabalho acabado; mediações editoriais; pressão sobre os revisores para que completem seus comentários em tempo mais curto; suscetibilidades das partes às intervenções e decisões, etc. Não obstante, essa tarefa fundamental é um requisito de boa qualidade, sem a qual todas as pretensões de progresso e crescimento de um veículo de publicação ficam comprometidas. Sem ela não se pode sequer considerar que uma revista seja "científica".

Pois então, apesar das dificuldades, é nessa condição que se deve investir. Começando pelos agradecimentos dos editores a essas pessoas que, verdadeiramente, constituem os alicerces da revista e de cujas contribuições ela não pode prescindir: os membros de nosso Conselho Editorial e todos os outros que, por nossas necessidades, foram chamados a colaborar. Sem fazer distinções de suas participações, mais (ou menos) elaboradas, com maior (ou menor) presteza, mais (ou menos) elegantes; mas, principalmente, por suas disponibilidades demonstradas, nossos carinhosos agradecimentos. A todos. E pois, nesta oportunidade, nomeando aos que foram por nós convocados, adicionalmente, a essa árdua, responsável e produtiva tarefa:

Abelardo de Souza Couto Junior

Abraão Garcia Mendes

Acácio Alves de Souza Lima F

Acácio Muralha Neto

Adamo Lui Netto

Aderbal de Albuquerque Alves

Amaryllis Avakian

Ana Estela B. P. Ponce Sant'ana

Ana Lúcia Colella

Ana Rosa Pimentel de Figueiredo

Ana Tereza Ramos Moreira

Antonio Augusto Velasco e Cruz

Antonio Sérgio Petrilli

Augusto Paranhos Jr.

Carlos Akira Omi

Carlos Eduardo Leite Arieta

Carlos Filipe C. C. Chicani

Carlos Fumiaki Uesugui

Carlos Rubens Lucas de Figueiredo 
Carmo Mandia Junior

Cássio Galvão Monteiro

Célia Regina Nakanami

Celina Tamaki Monteiro de Castro

Christiane Regina Rolim de Moura

Christine Mae Morello Abbud

Cláudia Maria Francesconi

Cláudia Rocha Lauretti

Cláudio Luis M. Petrilli

Clélia Maria Erwenne

Cristina Maria Bittencourt Garrido

Daena Nascimento Barros Leal

Daniel Madeira

Danielle Britto Miranda Silva

Danilo Dimas Monteiro de Castro

Décio Brunoni

Denise Fornazari de Oliveira

Edison João Geraissate Filho

Edméa Rita Temporini Nastari

Ednei Graciano do Nascimento

Edson Procianoy

Eduardo Jorge Carneiro Soares

Eduardo Sone Soriano

Élcio Hideo Sato

Eliana Aparecida Forno Velasco

Epaminondas Castelo Branco Neto

Eurípedes da Mota Moura

Fabiana Paula Tambasco

Fabíola Pacifico Seabra

Fausto Uno

Felício Aristoteles da Silva

Fernando Cenci Guimarães

Fernando Kayat Avvad

Flávio Jaime da Rocha

Flávio Rocha Lima Paranhos

Francisco de Assis Cordeiro Barbosa

Francisco Eduardo Lopes de Lima

Gabriela Soares Corrêa Meyer

Hamilton Moreira

Hélcio Jose Fortuna Bessa

Hélia Soares Angotti

Ítalo Mundialino Marcon

Jacob Moyses Cohen

Jacobo Melamed Cattan

Jair Mari
João Amaro Ferrari Silva

João Antonio Prata Junior

João Francisco Centola Nóbrega

Joelice Santos de Araújo

José Álvaro Pereira Gomes

José Antonio de Almeida Milani

José Aparecido da Silva

José Guilherme de C. Pecego

José Onildo Bertioli Contel

José Paulo Cabral Vasconcellos

José Ricardo de A. Reggi

José Vital Filho

José Wilson Cursino

Joyce Hisae Yamamoto

Juliana Maria Ferraz Sallum

Keila Míriam M. de Carvalho

Kozo Nakano

Laudo Silva Costa

Leiria de Andrade Neto

Liana Maria V. de O. Ventura

Lucia Miriam Dumont Lucci

Luciene Barbosa de Souza

Luís Carlos Ferreira de $\mathrm{Sa}$

Luís Eduardo Morato R.de Carvalho

Luís Paves

Magno Antonio Ferreira

Marcelo Carvalho Ventura

Márcia Motono

Marcia Regina Issa Salomão Libânio

Marcio Boaventura Maia

Marcos Carvalho da Cunha

Marcos Wilson Sampaio

Maria Auxiliadora M. F. Sibinelli

Maria Carmen Menezes Santos Torres

Maria Cristina Nishiwaki Dantas

Maria de Lourdes M.M.Villas Boas

Marivaldo Castro de Oliveira

Marizilda Rita de Andrade Giovedi

Marta Beatriz C. F. Sartori

Marta Junqueira Henriques Borges

Maurício Bastos Pereira

Mauro Albuquerque

Mauro Goldbaum

Mauro Nishi

Michel Eid Farah Neto
Miguel Ângelo Padilha Velasco

Mônica Fialho Cronemberger

Mônica Rinkevicius

Newton Kara José Jr.

Nilva Simeren Bueno Moraes

Nivaldo Vieira de Souza

Norma Allemann

Norma Helen Medina

Paulo César Silva Fontes

Paulo Elias Correa Dantas

Paulo Góis Manso

Paulo Henrique de Ávila Morales

Paulo Ricardo de Oliveira

Paulo Schor

Paulo Sérgio Moraes Barros

Procópio Miguel dos Santos

Regiani Lopes Malicia Bauzys

Regina Halfeld Furtado de Mendonça

Renato Giovedi Filho

Renato Luiz Gonzaga

Ricardo Belfort

Ricardo Morschbacher

Ricardo Themudo Lessa Waetge

Ricardo Uras

Roberto Caldato

Roberto Freire Santiago Malta

Rodrigo Jorge

Rosalia Maria Simões A. Foschini

Rosana Nogueira Pires da Cunha

Rosane da Cruz Ferreira

Ruth Miyuki Santo

Seiji Hayashi

Sidney Júlio de F. e Sousa

Silvana Artioli Schellini

Sílvia Veitzman

Simone H. D. von Faber Bison

Solange Rios Salomão

Sueli de Faria Müller

Suzana Matayoshi

Teruo Aiahara

Tomás F. Scalamandré Mendonça

Wagner Zacharias

Walter Yukihiko Takahashi

Walton Nosé

Wesley Ribeiro Campos

Especialmente, sobretudo, aos nossos queridos Conselheiros Editoriais que enriqueceram o prestígio dos Arquivos Brasileiros de Oftalmologia emprestando-lhes o prestígio dos seus; que conservaram suas dedicações para com nossa revista, tomando parcelas importantes de seus tempos para priorizá-la, oferecendo comentários construtivos em suas análises; e que agora, temporariamente, cedem seus postos na renovação anual dos quadros de nossos colaboradores. 